\title{
Erratum to: Rapid Determination of Mercury in Contaminated Soil and Plant Samples Using Portable Mercury Direct Analyzer Without Sample Preparation, a Comparative Study
}

\author{
John G. Kelly • Fengxiang X. Han • Yi Su • \\ Yunjun Xia • Valerie Philips • Zhiqi Shi • \\ David L. Monts • Sergio T. Pichardo • Kang Xia
}

Published online: 7 June 2014

(C) Springer International Publishing Switzerland 2014

\section{Erratum to: Water Air Soil Pollut DOI 10.1007/s11270-011-1030-3}

After publication, the authors noticed some typographical errors in Table 3. Time per sample should be "min". LOD of ICP-AES and CVAAS should be 0.480 and $0.048 \mathrm{ppb}$, respectively, while LOQ of ICP-AES and CVAAS should be 1.60 and 0.160 ppb, respectively.

The information is correct within the body of the text. The corrected table appears below.

The authors apologize for this oversight.
Table 3 Comparison of some relevant parameters of three instruments for $\mathrm{Hg}$ detection in soil and plant samples

\begin{tabular}{llll}
\hline Item & ICP-AES & CVAAS & RA-915+ \\
\hline Sample preparation & Yes & Yes & Yes \\
Time per sample (min) & 5 & 2 & 1 \\
LOD (ppb) & 0.48 & 0.048 & 3.36 \\
LOQ (ppb) & 1.60 & 0.160 & 11.1 \\
Precision (\%) & 3.36 & 3.73 & 3.09 \\
Accuracy & $84.4 \pm 3.49$ & $102 \pm 4.50$ & $91.6 \pm 3.60$
\end{tabular}

The online version of the original article can be found under http:// dx.doi.org/10.1007/s11270-011-1030-3.

J. G. Kelly • F. X. Han • Y. Su • Y. Xia • V. Philips •

S. T. Pichardo Institute for Clean Energy Technology,

Mississippi State University,

205 Research Blvd.,

Starkville, MS 39759, USA

F. X. Han $(\bowtie)$

Department of Chemistry and Biochemistry,

Jackson State University,

1400 J. R. Lynch St.,

Jackson, MS 39217, USA

e-mail: han@icet.msstate.edu
Z. Shi

Institute of Food Quality, Safety and Monitoring,

Jiangsu Academy of Agricultural Sciences,

Nanjing 210014, China

D. L. Monts

Department of Physics and Astronomy, Mississippi State

University,

Mississippi State, MS 39762, USA

K. Xia

Department of Crop and Soil Environmental Science,

Virginia Polytechnic Institute and State University,

Blacksburg, USA 